\title{
Administración de medicamentos sin indicación médica en menores de cinco años de una ciudad del norte del Perú
}

\author{
Cristhian Marcelo de Jesús Brenis-Díaz 1,a, Milagros Sarahí Marcelo De los Santos 1,a, \\ Andrea Bertha Rojas-Rioja 1,a, Sebastian Iglesias-Osores ${ }^{2, b}$, Zhandra Arce-Gil 1,c
}

\section{RESUMEN}

Objetivo. Caracterizar la administración de medicamentos sin indicación médica en menores de cinco años en la ciudad de Chiclayo, Lambayeque. Material y métodos. Estudio descriptivo transversal con muestreo no probabilístico consecutivo realizado en dos centros comerciales. Se aplicó un cuestionario validado por expertos. Resultados. Se entrevistó a 379 madres. El promedio de edad fue de 30,49 $\pm 8,29$ años, mediana de 29; (rango 25-75: 18 a 70 años). La frecuencia de medicación por parte de los padres hacia sus hijos con antibióticos fue el 70,7 \%. El antibiótico más usado fue amoxicilina (33,9\%). La manifestación clínica más frecuente descrita por las madres encuestadas fue el dolor de garganta y resfrío; y la fiebre fue el signo clínico más frecuente para el uso de antibióticos. El motivo más frecuente para no acudir al médico fue: "Ya sé lo que recetan". Ser conviviente se asoció a automedicación. Conclusiones. La frecuencia de automedicación en niños menores de cinco años del distrito de Chiclayo fue elevada. El fármaco más prescrito fue amoxicilina y el motivo más frecuente para no acudir a un médico fue que las madres ya sabían lo que recetan.

Palabras Clave: Automedicación, Antibióticos, Infantes (Fuente: DeCS-BIREME)

\section{Administration of medications without medical indication in children under five years of a city in northern Peru}

\begin{abstract}
Objective. Characterize the administration of medications without medical indication in children under five in the city of Chiclayo, Lambayeque.Material and methods. Descriptive cross-sectional study with consecutive non-probabilistic sampling conducted in two shopping centers. A questionnaire validated by experts was applied. Results. 379 mothers were interviewed. The average age was $30.49 \pm 8.29$ years, median of 29; (range 25-75: 18 to 70 years). The frequency of medication by parents to their children with antibiotics was $70.7 \%$. The most commonly used antibiotic was amoxicillin (33.9\%). The most frequent clinical manifestation described by the surveyed mothers was a sore throat and a cold; and fever was the most frequent clinical sign for antibiotic use. The most frequent reason for not seeing a doctor was: "I know what they prescribe." Being a partner was associated with self-medication. Conclusions. The frequency of self-medication in children under five in the Chiclayo district was high. The most prescribed drug was amoxicillin and the most frequent reason for not seeing a doctor was that mothers already knew what they prescribed.
\end{abstract}

Keywords: Self-medication, Antibiotics, Infants (Source: MeSH-NLM)

\footnotetext{
${ }^{1}$ Facultad de Medicina Humana, Universidad Católica Santo Toribio de Mogrovejo. Chiclayo, Perú.

2 Hospital Regional Lambayeque. Chiclayo, Perú.

a Estudiante de Medicina Humana..

${ }^{\mathrm{b}}$ Biólogo

c Biólogo, Magíster en Microbiología.
} 


\section{INTRODUCCIÓN}

La Organización Mundial de la Salud (OMS) ha definido automedicación como "el uso de medicamentos para tra-tamientos de trastornos o síntomas autorreconocidos"(1). La práctica habitual de la automedicación sin responsabilidad, la convierte en un problema actual de salud pública, debi-do al riesgo que supone para las personas y al aumento de emergencias extrahospitalarias y la resistencia bacteriana (2). Por otra parte, no solo provoca una alteración en el curso normal de la enfermedad, si no también afecta la capacidad diagnóstica, el proceso de recuperación y puede ocasionar intoxicaciones por el uso inadecuado de estos ${ }^{(3)}$.

En Perú, la prevalencia de automedicación en la población varía entre 40 y $60 \%{ }^{(4)}$, en Cajamarca por ejemplo se mostró que la automedicación es un problema frecuente en zonas rurales, donde está presente en el $36,19 \%$ de los hogares ${ }^{(5)}$.

Una de las poblaciones más vulnerables son los niños, en especial los menores de cinco años pues tienen mayor ries-go de contraer enfermedades o adquirir resistencia a antibió-ticos con mayor facilidad en caso de automedicación (6). Las personas responsables del cuidado de los niños son mayor-mente las madres, siendo el fenómeno de automedicación frecuente en ellas (7).

Analizar esta situación es de vital importancia, puesto que permitirá conocer percepciones relacionadas con esta pro-blemática, así como la obtención de datos estadísticos nece-sarios sobre automedicación de los padres con antibióticos en niños. El objetivo del estudio es caracterizar la adminis-tración de medicamentos sin indicación médica en menores de cinco años en Chiclayo, Perú, durante el 2017.

\section{MATERIAL Y MÉTODOS}

\section{Tipo y diseño de investigación}

El estudio descriptivo transversal.

\section{Población y muestra}

El estudio se llevó a cabo en madres de infantes de la ciudad de Chiclayo, departamento de Lambayeque, al Norte del Perú. El tamaño de muestra se estimó a una frecuencia del 50\% de una población de 25067 niños menores de cinco años (INEI), con un nivel de confianza de 95\%; una precisión del $5 \%$ y un efecto de diseño de 1 . Se calculó un tamaño muestral de 379 madres, empleando OpenEpi. El muestreo fue no probabilístico consecutivo hasta alcanzar el tamaño de muestra esperado utilizando como instrumento una en-cuesta que se aplicó a las madres de los niños menores de cinco años en un centro comercial y en un mercado mayoris-ta del distrito de Chiclayo.

Los criterios de inclusión fueron: radicar actualmente en el distrito de Chiclayo, firma del consentimiento informado y au-sencia de deterioro cognitivo.

\section{Instrumentos de recolección de datos}

Para la recolección de datos se utilizó un cuestionario estructurado y compuesto por dos secciones: datos sociodemográficos y diez preguntas cerradas de alternativa múltiple. Cada pregunta se calificó de forma independiente.

\section{Análisis de datos}

Se usó estadística descriptiva. Los datos fueron procesados usando una hoja de cálculo de Microsoft Excel y empleando el complemento de MegaStat para la estadística descriptiva. Se hizo un análisis exploratorio bivariado entre las características sociodemográficas de las madres y las preguntas del cuestionario.

\section{Consideraciones éticas.}

El proyecto fue aprobado por el Comité de Ética de la Facultad de Medicina de la Universidad Católica Santo Toribio de Mogrovejo. La participación fue voluntaria mediante la firma de un consentimiento informado.

\section{RESULTADOS}

Se encuestaron 379 madres de niños que respondieron al cuestionario. El promedio de edad fue de 30,5 $\pm 8,3$ años; (rango: 18 a 70 años); los datos sociodemográficos se describen en la Tabla 1.

Tabla 1. Características sociodemográficas de las madres de menores de cinco años de una ciudad del norte del Perú.

\begin{tabular}{|c|c|c|c|c|c|}
\hline \multirow{2}{*}{ Variables } & \multicolumn{2}{|c|}{ Sí } & \multicolumn{2}{|c|}{ No* } & \multirow{2}{*}{ Valor $p$} \\
\hline & $\mathbf{N}$ & $\%$ & $\mathbf{N}$ & $\%$ & \\
\hline \multicolumn{6}{|l|}{ Grado de instrucción } \\
\hline $\begin{array}{l}\text { Sin instrucción y } \\
\text { primaria }\end{array}$ & 25 & 9,4 & 14 & 13,2 & \multirow{2}{*}{0,18} \\
\hline Secundaria y superior & 240 & 90,6 & 92 & 86,8 & \\
\hline \multicolumn{6}{|l|}{ Lugar de encuesta } \\
\hline Mercado de abastos & 242 & 90,3 & 97 & 87,4 & \multirow{2}{*}{0,25} \\
\hline Centro comercial & 26 & 9,7 & 14 & 12,6 & \\
\hline \multicolumn{6}{|l|}{ Estado civil } \\
\hline Soltera & 55 & 20,8 & 22 & 20,8 & \\
\hline Conviviente & 165 & 62,3 & 50 & 47,2 & \\
\hline Casada & 41 & 15,5 & 31 & 29,3 & 0,03 \\
\hline Divorciada & 2 & 0,8 & 1 & 0,9 & \\
\hline Viuda & 2 & 0,8 & 2 & 1,9 & \\
\hline Edad (media \pm DS) & \multicolumn{2}{|c|}{$30,49 \pm 8$} & \multicolumn{2}{|c|}{$30,5 \pm 9,2$} & 0,98 \\
\hline $\begin{array}{l}\text { Número de hijos } \\
\text { (media } \pm D S)\end{array}$ & \multicolumn{2}{|c|}{$2,05 \pm 1,1$} & \multicolumn{2}{|c|}{$2,16 \pm 1,4$} & 0,99 \\
\hline
\end{tabular}

* Incluye no automedicación y automedicación con otros fármacos.

** DS=Desviación estándar

Del total de madres encuestadas, 297 (78,3\%) habían medicado alguna vez a sus hijos, $268(90,2 \%)$ lo hicieron con antibióticos. 
Por lo tanto, del total de madres encuestadas, el 70,7 \% había automedicado con antibióticos alguna vez a sus hijos. El $62,3 \%$ de las madres convivientes automedicaban a sus hijos, hallándose asociación significativa $(p=0,03)$. La edad, el grado de instrucción, el lugar de toma de datos y el número de hijos, no se asociaron a automedicación. El medicamento usado con más frecuencia fue amoxicilina: 33,9 $\%$ (91/268), seguido de trimetropim/sulfametoxazol: 5,9\% (16/268). Según la frecuencia de automedicación, el 34,3\% madres (93/268) administraban tres dosis del antibiótico al día; $47 \%$ (126/268) dos veces al día; 17,2 \% (46/268) una vez al día y $1,1 \%(3 / 268)$ solo una dosis (Tabla 2$)$.

El promedio del número de días de prescripción de antibióticos fue tres; ninguna de las madres dio el antibiótico por más de siete días; $117(43,6 \%)$ administraban el antibiótico por tres días, 147 (54,8\%) utilizó el mismo antibiótico en dos oportunidades y 49 (18,3\%) por cinco días; en los últimos siete meses.

Los motivos más frecuentes de automedicación fueron: "ya sé lo que recetan" (53,4 \%), "por recomendación de un familiar o compañeros" (23,1\%), "falta de dinero para la consulta" (11,19\%); "falta de tiempo para acudir al médico (10,8 \%)"; y "no lo había pensado (1,5\%)". Del total de madres, $(68,3 \%)$ manifestaron que la automedicación podría generar algún daño en sus hijos.

Tabla 2. Características de medicación según esquema y tipo de antibiótico más usado menores de cinco años de una ciudad del norte del Perú.

\begin{tabular}{lcc} 
Características de la administración de medi- & N & $\%$ \\
camentos & & \\
Esquemas más utilizados & 91 & 34,0 \\
Amoxicilina & 69 & 25,8 \\
Amoxicilina/Trimetropim-Sulfametoxazol & 16 & 6,0 \\
Trimetropim-Sulfametoxazol & 11 & 4,1 \\
Amoxicilina/ Trimetropim-Sulfametoxazol /Eritro- & & \\
micina & 7 & 2,6 \\
Amoxicilina/Eritromicina & 7 & 2,6 \\
Amoxicilina/Furazolidona & 7 & 2,6 \\
Amoxicilina/ Trimetropim-Sulfametoxazol /Azitro- & & \\
micina & 6 & 2,2 \\
Amoxicilina/ Trimetropim-Sulfametoxazol /Fura- & 6,2 \\
zolidona & 5 & 1,9 \\
Amoxicilina+Ac. Clavulánico & 59 & 18,3 \\
Otros & 49 \\
Antibióticos más utilizados & & \\
Amoxicilina & 185 & 69,0 \\
Trimetropim-Sulfametoxazol & 56 & 20,9 \\
Furazolidona & 8 & 3,0 \\
Azitromicina & 8 & 3,0 \\
Eritromicina & 7 & 2,6 \\
Amoxicilina + Ácido clavulanico & 3 & 1,1 \\
Claritromicina & & \\
\hline \hline
\end{tabular}

Según los grupos por los que las madres automedicaban encontramos: "dolor de garganta + resfrío" $27,9 \%$, "dolor estomacal + diarrea" 21,3\%, "fiebre" $15,1 \%$, "fiebre + dolor de garganta + resfrío" $13,1 \%$, "fiebre + dolor de estomacal + diarrea + dolor de garganta + resfrío" $6,4 \%$, otra molestia $4 \%$, dolor de cabeza $3,4 \%$, otros síntomas $8,8 \%$.

\section{DISCUSIÓN}

La medicación no es una decisión autónoma, libre y voluntaria en el caso de los niños. Por el contrario, en este caso se basa en la interpretación subjetiva de los síntomas realizada por la madre, aunque pocas investigaciones han estudiado aspectos de la automedicación en niños y adolescentes, algunos autores han informado una alta prevalencia de automedicación en este grupo de edad (8).

En nuestro estudio del total de madres, más de la mitad habían medicado alguna vez a sus hijos, de las cuales más de tres tercios lo hicieron con antibióticos. Cifras similares se encontraron en un estudio realizado en Nigeria por Akinlade et al. en el que el que se usaban antibióti$\cos { }^{(7)}$. Ecker en Lima, en su estudio hallaron que de niños menores de cinco años recibieron antibióticos ${ }^{(6)}$.

El medicamento más usado en nuestro estudio fue amoxicilina, similar a diversos estudios en el que también el antibiótico de uso más frecuente es la Amoxicilina (9-11), seguido de Trimetropim-Sulfametoxazol similar a otro estudio en el que junto con la amoxicilina y amoxicilina-clavulánico representaron el $85 \%$ de los antibióticos usados (12). Según la frecuencia de automedicación en nuestro estudio, el $34,33 \%$ madres administraban tres dosis del antibiótico al día; $47,01 \%$ dos veces al día; $17,16 \%$ una vez al día y $1,12 \%$ solo una dosis, en otro estudio se encontró el uso de dosis insuficiente en la automedicación que corrobora los datos de unidosis dadas por las madres a sus hijos en nuestro estudio ${ }^{(13)}$. El promedio del número de días de prescripción de antibióticos fue tres; ninguna de las madres dio el antibiótico por más de siete días; $117(43,6 \%)$ administraban el antibiótico por tres días y $49(18,28 \%)$ por cinco días; 147 (54,8 \%) utilizó el mismo antibiótico en dos oportunidades en los últimos siete meses, esto es similar a otros estudios en la que la práctica inapropiada más común en el uso agentes antimicrobianos que incluye corta duración del tratamiento, en su mayoría menos de cinco días ${ }^{(14,15)}$.

El motivo más frecuente por el que las madres automedicaban a sus hijos y no lo llevaban al médico era que referían saber qué es lo que se receta. Del total de madres, $183(68,28 \%)$ manifestaron que la automedicación podría generar algún daño en sus hijos, en otro estudio los motivos para la medicación fueron ahorro de tiempo, ahorro de dinero, funciono con familiares, etc. (16). En 
el estudio de Ecker, la mayoría adquirió los antibióticos sin prescripción médica, debido a que la atención médica demora mucho $(21,3 \%)$ y creían que era un caso leve por lo que no requerían de atención médica (18\%)(6). En contraste con nuestro estudio donde se encontró que las madres no llevaron a sus hijos al médico por motivos de "falta de tiempo": 29 (10,82\%); seguido "por recomendación de un familiar o compañeros": $62(23,13 \%)^{(6)}$.

La automedicación de las madres hacia sus hijos se da también con analgésicos de venta libre se asocia, predominantemente paracetamol, entre los niños en edad escolar (8); lo cual no incluimos en nuestro estudio pero es importante conocer. Se encontraron similitudes con nuestro estudio con el de Alarco en el que tener "Dolor estomacal + diarrea" las madres medicaban a sus hijos dar antibióticos (17). Ecker halló que el cuadro clínico más frecuente para el cual se usaban antibióticos era el "resfrío". En nuestro estudio los resultados fueron semejantes, los cuadros clínicos más frecuentes fueron: "resfrío y dolor de garganta". Estas manifestaciones obedecen en su mayoría a cuadros virales para los que no se necesitan antibióticos. Asimismo, el síntoma más frecuente descrito por las madres fue "fiebre" $(13,4 \%)$ resultado que coincide con el estudio de Zevallos realizado en Dakar Senegal (18). Una probabilidad es que las características de la automedicación sean similares en estas tres realidades.

Llama la atención que las madres encuestadas en nuestro estudio referían que el uso de antibióticos les puede causar algún daño y sin embargo lo seguían haciendo, además de causar posible resistencia bacteriana, que al no tener supervisión médica el uso de dosis no recomendadas o por debajo del estándar es frecuente. La resistencia bacteriana se ha convertido en un grave problema de salud en los últimos años debido al uso indiscriminado de antibióticos y causa muchas muertes por año (19-20). Posiblemente esto se deba a la falta de información sobre las consecuencias en la salud del uso de antibióticos sin prescripción médica ${ }^{(21)}$. La falta de información sanitaria, desempleo, facilidad para conseguir medicamentos, dificultad para obtener consultas médicas produciendo alteración de la práctica bajo el concepto de "automedicación responsable" lo que ha convertido en un problema frecuente de salud pública que se presenta en personas de todo grado de instrucción ${ }^{222}$. En nuestra opinión, analizar esta situación es de gran importancia para conocer los factores determinantes, las percepciones y las realidades relacionadas con este problema.

Este problema debe ser abordado por las instituciones en salud para que se pueda realizar un cambio y aproximarse al autocuidado responsable aplicando ciertas medidas como mejorar el sistema de atención de los centros de salud, brindando información sobre los efectos adversos que producen los antibióticos en sus niños, supervisar la publicidad audiovisual descontrolada que incentiva al uso irresponsable anti- bióticos y mejorar la educación en salud de la población con charlas informativas creando conciencia sobre las consecuencias de la automedicación irresponsable en la salud comunitaria.

Las limitaciones de nuestro estudio fueron que el instrumento de evaluación fue revisado por expertos, más no validado; sin embargo, se realizó una prueba piloto para medir su nivel de entendimiento. Hubo rechazo por parte de un grupo de madres, por lo que se completó el tamaño de muestra con madres del otro centro comercial. Las principales fortalezas fueron que se evitó el sesgo de memoria mediante el uso de una cartilla con la imagen de cada uno de los medicamentos que se utilizó en el instrumento de evaluación.

En conclusión, la frecuencia de automedicación en niños menores de cinco años del distrito de Chiclayo es elevada. El fármaco más prescrito fue amoxicilina y el motivo más frecuente para no acudir a un médico fue que las madres ya sabían lo que recetan.

\section{REFERENCIAS BIBLIOGRÁFICAS}

1. Vera-Romero OE, Urcia-Peláez JMM, Ayala-Bravo E, Falla-Aldana BS, Díaz-Vélez C. La Automedicación en los estudiantes de la Universidad Nacional de la Región Lambayeque durante el periodo Noviembre 2010 - Diciembre 2012. Rev del Cuerpo Médico del HNAAA. 2016;9(1):20-31.

2. Zapata Fajardo LA. La automedicación de antibióticos: un problema de salud pública. Rev Científica Salud Uninorte. 2013;29(2): 226-35.

3. Vera Romero O, Rodas Regalado C, Falla Aldana BS. La Automedicación: una problemática nacional y regional que se debe prevenir. Rev del Cuerpo Médico Hosp Nac Almanzor Aguinaga Asenjo. 2011; 4(2):129-31.

4. Pillaca Medina ML, Carrión Domínguez K, Domínguez KC. Automedicación en personas adultas que acuden a boticas del Distrito Jesús Nazareno, Ayacucho 2015. An la Fac Med. 2016; 77(4):387.

5. Llanos Zavalaga LF, Contreras Ríos CE, Velásquez Hurtado JE, Mayca Pérez JA, Lecca García L, Reyes Lecca R, et al. Automedicación en cinco provincias de Cajamarca. Rev Medica Hered. 2001; 12(4):127-33.

6. Ecker L, Ruiz J, Vargas M, Del Valle LJ, Ochoa TJ. Prevalence of purchase of antibiotics without prescription and antibiotic recommendation practices for children under five years of age in private pharmacies in Periurban areas of Lima, Peru. Rev Peru Med Exp Salud Publica. 2016; 33(2):215-23.

7. Akinlade KA, Akinyemi JO, Fawole OI. Knowledge of hazards of antibiotics self-medication by mothers for under-fives in rural community of South-west Nigeria. Afr J Med Med Sci. 2015; 44(4):303-9.

8. Valenzuela Ortiz M, Sánchez Ruiz-Cabello FJ, Uberos J, Checa Ros AF, Valenzuela Ortiz C, Augustín Morales MC, et al. Automedicación, autoprescripción y medicación «por poderes» en pediatría. An Pediatría. 2017; 86(5):264-9.

9. Miní E, Varas R, Vicuña $Y$, Lévano M, Rojas L, Medina J, et al. [Self-medication behavior among pregnant women user of the Instituto Nacional Materno Perinatal, Peru 2011]. Rev Peru Med Exp Salud Publica. 2012; 29(2):212-7.

10. Barber DA, Casquejo E, Ybañez PL, Pinote MT, Casquejo L, Pinote LS, et al. Prevalence and correlates of antibiotic sharing in the Philippines: antibiotic misconceptions and community-level access to non-medical sources of antibiotics. Trop Med Int Heal. 2017; 22(5):567-75.

11. Moise K, Bernard JJ, Henrys JH. Evaluation of antibiotic self-medication among outpatients of the state university hospital of Port-Au-Prin- 
ce, Haiti: a cross-sectional study. Pan Afr Med J. 2017;28:4.

12. Mohanna M. Self-medication with Antibiotic in Children in Sana'a City, Yemen. Oman Med J. 2010; 25(1):41-3.

13. Sihavong A, Lundborg CS, Syhakhang L, Akkhavong K, Tomson G, Wahlstrom R. Antimicrobial self medication for reproductive tract infections in two provinces in Lao People's Democratic Republic. Sex Transm Infect. 2006; 82(2):182-6.

14. Widayati A, Suryawati S, De Crespigny C, Hiller JE. Self medication with antibiotics in Yogyakarta City Indonesia: A cross sectional population-based survey. BMC Res Notes. 2011; 4(1):491.

15. Onanuga A, Temedie TC. Multidrug-resistant Intestinal Staphylococcus aureus among Self-medicated Healthy Adults in Amassoma, South-South, Nigeria. J Heal Popul Nutr. 2011; 29(5):446-53.

16. Shah SJ, Ahmad H, Rehan RB, Najeeb S, Mumtaz M, Jilani MH, et al. Self-medication with antibiotics among non-medical university students of Karachi: a cross-sectional study. BMC Pharmacol Toxicol. 2014; 15(1):74.

17. Jhonnel Alarco J, Aguirre-Cuadros E, Alvarez-Andrade E V. Conocimiento de las madres sobre la diarrea y su prevención en un asentamiento humano de la provincia de Ica, Perú. Rev Clínica Med Fam. 2013; 6(1):25-31.

18. Ndiaye P, Tal-Dia A, Diedhiou A, Juergens-Behr A, Lemort JP. [Self-treatment of fever in the northern district of Dakar, Senegal]. Med Trop (Mars). 2006; 66(1):74-8.

19. Munita JM, Arias CA. Mechanisms of Antibiotic Resistance. In: Virulence Mechanisms of Bacterial Pathogens, Fifth Edition. American Society of Microbiology; 2016. p. 481-511.

20. Watkins RR, Bonomo RA. Overview: Global and Local Impact of Antibiotic Resistance. Infect Dis Clin North Am. 2016; 30(2):313-22.

21. You M-A, Nam S-M, Son Y-J. Parental Experiences of Medication Administration to Children at Home and Understanding of Adverse Drug Events. J Nurs Res. 2015; 23(3):189-96.

22. Zhao Y, Ma S. Observations on the Prevalence, Characteristics, and Effects of Self-Treatment. Front Public Heal. 2016;4: 69. 